Palavras chave: Madeira Colorimetria Cetol Verniz Intemperismo

Histórico: Recebido 03/09/2014 Aceito 07/03/2016

Keywords: Wood Colorimetry Cetol Varnish Weatherin

Correspondência: jacob.thais@gmail.com
Thais Jacob Mendes', Joaquim Carlos Gonçalez², Ricardo Faustino Teles', Clarissa Melo Lima'

\section{EFEITO DO INTEMPERISMO ARTIFICIAL NA COR DE LÂMINAS DE MADEIRA TRATADAS COM DOIS PRODUTOS DE ACABAMENTO.}

RESUMO: O intemperismo é um dos principais motivos da deterioração da madeira, principalmente de sua cor. A aplicação de produtos de acabamento minimiza esses efeitos. Este trabalho teve como objetivo avaliar o efeito do intemperismo artificial sobre lâminas de madeira das espécies de cumaru (Dipteryx odorata) e pau marfim (Balfourodendron riedelianum) tratadas com dois produtos de acabamento com monitoramento por meio de um espectrofotômetro. As amostras foram submetidas a ciclos de exposição ao intemperismo de 20, 40, 52, 76, 124, 226, 430, 838 e 960 horas. Os parâmetros colorimétricos ( $\mathrm{L}^{*}, \mathrm{a}^{*}, \mathrm{~b}^{*}, \mathrm{C}$ e h*) foram medidos antes dos tratamentos, após a aplicação dos produtos e durante os intervalos de tempo de intemperismo. A aplicação dos produtos de acabamento escureceu as lâminas de madeiras de cumaru e de pau marfim em estado natural. No entanto, nos tempos de intemperismos mais elevados, ambas as espécies voltaram a clarear, chegando mesmo a serem superiores os valores da claridade ao da madeira natural. $O$ uso do cetol se mostrou mais eficiente, dando maior estabilidade na conservação da cor da madeira das espécies estudadas.

\section{EFFECT OF ARTIFICIAL WEATHERING ON WOOD LAMINATES COLOR TREATED WITH TWO FINISHING PRODUCTS.}

ABSTRACT: Weathering is one of the main reasons for the degradation of wood, especially its color. The application of finishes minimizes these effects. This study aimed to monitor the effect of artificial weathering on wood veneer of the species cumaru (Dipteryx odorata) and pau marfim (Balfourodendron riedelianum) with two finishes, the marine varnish and Cetol, with monitoring using a spectrophotometer. The samples were subjected to cycles of exposure to weathering for $20,40,52,76,124,226,430$, 838 and 960 hours. The colorimetric parameters ( $\mathrm{L}^{*}, \mathrm{a}^{*}, \mathrm{~b}^{*}, \mathrm{C}$ and $\mathrm{h}^{*}$ ) were measured before treatment, after application of the products and during the weathering time intervals. The application of finishes darkened veneer of cumaru wood and pau marfim in nature. However, in higher weathering times, both species returned to a lighter color, and even became lighter than the natural wood. The use of Cetol was more efficient, giving greater stability in the conservation of wood color of the species studied. 


\section{INTRODUÇÃO}

Entre os materiais construtivos, a madeira destaca-se como um material versátil, com aplicabilidade diversificada de usos. No entanto, a maioria das espécies florestais é suscetível a intempéries, alterando suas características naturais, podendo mesmo inviabilizar sua utilização.

O uso da madeira ou de suas lâminas para fabricação de móveis, de assoalhos, ou de outras formas de utilização interiores é determinado, principalmente, pelo seu aspecto, pela sua aparência superficial e em particular por sua cor. Sendo assim, a cor é um fator preponderante no mercado da madeira, muitas vezes, determinando o seu preço final (GONÇALEZ, 1993). O controle da mudança de coloração das lâminas gera produtos de qualidade mais homogêneos, agregando assim valor às peças.

Por outro lado, a cor da madeira é muito instável, estando sujeita a rápidas alterações. Essa propriedade pode ser alterada por ação do intemperismo, que é uma ação complexa e combinada do sol, da chuva ou umidade e dos ventos (HON, 200I). Assim, sempre que sujeitada à ação de intempéries, a cor e a textura da madeira são alteradas (SUDIYANI et al., 1999). Essas alterações ocorrem principalmente, devido à ação dos raios ultravioletas, que provocam tanto a fotodegradação quanto a fotodescoloração da madeira, diminuindo sua vida útil (GOUVEIA, 2008).

Segundo Silva e Pastore (2004) apesar da ação do intemperismo ser limitada à superfície da madeira, constitui um problema para os consumidores e para os tecnologistas, pois afeta o desempenho dos produtos aplicados sobre ela. Há diversas formas de prevenir à madeira da fotodegradação, como a aplicação de compostos químicos inorgânicos, a aplicação de produtos de acabamento, como vernizes, stains e tintas, que embora forneçam algum tipo de proteção, podem modificar o aspecto natural da madeira (GONÇALEZ et al., 20l0).

Segundo informações do manual do fabricante, - verniz marítimo acetinado é um polímero alquídico modificado, composto por óleos vegetais, hidrocarbonetos aromáticos, hidrocarbonetos alifáticos, agentes tensoativos, secantes organo metálicos e máximo $0,1 \%$ em volume de benzeno. $\mathrm{O}$ cetol acetinado natural é um produto à base de polímeros acrílicos em emulsão, pigmentos transparentes absorvedores de raios UV, bactericida e fungicida não metálico, agente fosqueante (na versão acetinada), aditivos e água.

Esta pesquisa teve como objetivo acompanhar o efeito do intemperismo artificial sobre lâminas de madeira das espécies de cumaru e pau marfim tratadas com dois produtos de acabamento (cetol e verniz marítimo) por meio da avaliação colorimétrica.

\section{MATERIAL E MÉTODOS}

Esta pesquisa foi desenvolvida no Laboratório de Tecnologia da Madeira do Departamento de Engenharia Florestal - UnB. As lâminas de madeira das espécies de cumaru (Dipteryx odorata) e pau marfim (Balfourodendron riedelianum) foram adquiridas no mercado madeireiro do Distrito Federal e apresentavam dimensões iniciais de $250 \times 14 \times 0,1 \mathrm{~cm}$.

Para o estudo foram utilizadas 12 amostras da espécie cumaru e 12 da espécie pau marfim nas dimensões de $50 \times 80 \mathrm{~mm}$, na forma de lâminas com espessura de I,0mm. As amostras foram levemente esfoliadas com lixas de granulometria 150 antes de receberem os produtos, incluindo as testemunhas, com a finalidade de uniformizar a superfície. Para cada tratamento foram utilizados quatro corpos de prova de cada espécie, além da testemunha. Os três tratamentos são: tratamento I - lâminas de madeira em estado natural (testemunha); tratamento 2 - verniz marítimo acetinado; tratamento 3 - cetol acetinado natural. A aplicação dos produtos foi feita com pincel, sendo aplicada duas demãos dos produtos em cada amostra.

A norma utilizada para a pesquisa de intemperismo artificial foi a ASTM G 154/2006, ciclo de exposição luminosa de 8 horas.

Os corpos de prova foram submetidos a testes que simulam os efeitos do intemperismo de forma acelerada, durante os períodos de: $20 \mathrm{~h}, 40 \mathrm{~h}, 52 \mathrm{~h}, 76 \mathrm{~h}$, I24h, 226h, 430h, 838h e 960horas.

Após o processo de acabamento foi feita a medição da cor no espectrofotômetro. As amostras de madeiras foram postas dentro da câmera de envelhecimento (QUV) da Q-Lab, modelo QUV/spray, para receber ciclos de radiação ultravioleta com o período inicial de 20 horas. Em seguida, as amostras foram retiradas da câmera e tomou-se a medida da cor. Este procedimento foi feito para todos os tempos mencionados até se completar - período de 960 horas. Cada amostra recebeu 180 leituras, sendo possível assim fazer uma varredura em toda a superfície da amostra (face tangencial).

A colorimetria (sistema CIELAB 1976) foi a técnica utilizada para acompanhamento do intemperismo. Os parâmetros colorimétricos de avaliação foram ( $L^{*}, a^{*}$, b*, C e h*). Onde o parâmetro $L^{*}$ indica luminosidade ou claridade, a* indica posição da cor sobre o eixo verdevermelho, b* a posição da cor sobre o eixo azul-amarelo, C a cromaticidade ou saturação e $h^{*}$ o ângulo de tinta, de acordo com o Sistema CIELab 1976 (GONCALEZ, I993). O espectrofotômetro utilizado foi o Color Eye XTH $X$-Rite, com iluminante D65 e ângulo de $10^{\circ} \mathrm{C}$ acoplado a um computador com o software Color iControl. Os parâmetros colorimétricos das amostras foram medidos antes das aplicações dos produtos de acabamento, após a aplicação dos produtos e a cada intervalo de tempo dos 
tratamentos na câmara de envelhecimento acelerado. Para mensurar a alteração na cor da madeira antes e após os tratamentos $(\Delta \mathrm{E})$ foi utilizada a Equação I, de acordo com a norma ASTM D 2244/2009.Em que: $\Delta \mathrm{L}$ é a variação do parâmetro $L^{*}$ entre as medições; $\Delta a$ é a variação do parâmetro $\mathrm{a}^{*}$ entre as medições; $\Delta \mathrm{b}$ é a variação do parâmetro b* entre as medições.

$$
\Delta \mathrm{E}=\sqrt{\Delta \mathrm{L}+\Delta \mathrm{a}^{2}+\Delta \mathrm{b}^{2}}
$$

Para verificar a variação total da cor $(\Delta \mathrm{E})$ em cada tratamento, utilizou-se a Tabela I, proposta por Hikita et al. (200I). Esta tabela está baseada em níveis de percepção, e faz uma classificação da variação total da $\operatorname{cor}(\Delta \mathrm{E})$ de madeiras.

Foi calculada a média de cada um dos parâmetros colorimétricos em cada medição, com a finalidade de verificar a existência de variação ao longo dos intervalos de exposição às intempéries através da análise de TABELA 1 Classificação da variação total da cor $(\Delta E)$ de madeiras. TABLE 1 Classification of the total variation $(\Delta E)$ of wood's color.

\begin{tabular}{cc}
\hline Variação da cor $(\Delta \mathrm{E})$ & Classificação \\
\hline $0,0-0,5$ & Desprezível \\
$0,5-1,5$ & Ligeiramente perceptível \\
$1,5-3,0$ & Notável \\
$3,0-6,0$ & Apreciável \\
$6,0-12,0$ & Muito apreciável \\
\hline
\end{tabular}

variância (ANOVA) e do teste de médias de Tukey a $5 \%$ de significância.

\section{RESULTADOS E DISCUSSÃO}

A Tabela 2 apresenta os valores médios dos parâmetros colorimétricos das amostras de lâminas de madeira cumaru e pau marfim antes e após receber os produtos de acabamento.

Analisando-se a Tabela 2, verifica-se que ao aplicar os produtos de acabamentos houve um escurecimento das lâminas de madeira das duas espécies, diminuindo-se os valores de $L^{*}$, aumentando os valores da variável $a^{*}$ e diminuindo os valores de $h^{*}$, quando comparado com a madeira das espécies testemunha. A redução de $h^{*}$ confirma o escurecimento da madeira, aproximando-as mais do eixo verde-vermelho no sistema CIEL*a*b.

Para o cumaru, entre os dois produtos de acabamento aplicados, observa-se que o verniz marítimo escureceu mais a madeira, pois o valor de L* é menor em relação às amostras tratadas com cetol. Essa característica pode ser notada também pela variável $h^{*}$ que posiciona as amostras tratadas com verniz marítimo mais próximo do eixo vermelho. No caso do pau marfim o escurecimento foi maior quando utilizado o cetol, o que também pode ser notado pela variável $h^{*}$. Considerando à coordenada $b^{*}$ para a madeira de cumaru constata-se uma diminuição para os dois tratamentos aplicados, o que sugere que os produtos possuem pigmento vermelho ou interagem

TABELA 2 Valores médios e desvio-padrão dos parâmetros colorimétricos para as amostras de madeira antes e após a aplicação do verniz marítimo e do cetol para as duas espécies estudadas.

TABLE 2 Mean values and standart deviation of colorimetric parameters of samples before and after treatment with verniz maritime and cetol for both species.

\begin{tabular}{|c|c|c|c|c|c|c|c|c|c|c|c|}
\hline \multirow[b]{2}{*}{ Tratamento } & \multirow[b]{2}{*}{ Estatística } & \multicolumn{5}{|c|}{ Madeira sem tratamento (natural) } & \multicolumn{5}{|c|}{ Madeira após aplicação dos produtos } \\
\hline & & $L^{*}$ & $a^{*}$ & $b^{*}$ & $\mathrm{C}$ & $h^{*}$ & $L^{*}$ & $a^{*}$ & $b^{*}$ & $\mathrm{C}$ & $\mathrm{h}^{*}$ \\
\hline \multicolumn{12}{|c|}{ Dipteryx odorata } \\
\hline \multirow[t]{2}{*}{ Testemunha } & Média & 49,09 & 10,04 & 23,66 & 25,70 & 67,00 & - & - & - & - & - \\
\hline & Desvio padrão & 1,59 & 0,48 & 1,06 & 1,11 & 0,77 & - & - & - & - & - \\
\hline \multirow[t]{2}{*}{ Verniz marítimo } & Média & 46,58 & 9,88 & 21,48 & 23,64 & 65,27 & 35,89 & 13,17 & 18,95 & 23,08 & 55,14 \\
\hline & Desvio padrão & 1,71 & 0,48 & 1,05 & 1,06 & 1,07 & 1,37 & 0,71 & 1,42 & 1,50 & 1,30 \\
\hline \multirow[t]{2}{*}{ Cetol } & Média & 48,71 & 10,18 & 23,50 & 25,61 & 66,59 & 42,18 & 11,19 & 21,71 & 24,43 & 63,25 \\
\hline & Desvio padrão & 1,62 & 0,51 & 1,40 & 1,45 & 0,84 & 4,82 & 1,46 & 2,63 & 2,94 & 4,97 \\
\hline \multicolumn{12}{|c|}{ Balfourodendron riedelianum } \\
\hline \multirow[t]{2}{*}{ Testemunha } & Média & 72,28 & 5,80 & 30,37 & 30,92 & 79,21 & - & - & - & - & - \\
\hline & Desvio padrão & 1,75 & 0,61 & 1,20 & 1,27 & 0,81 & - & - & - & - & - \\
\hline \multirow[t]{2}{*}{ Verniz marítimo } & Média & 72,46 & 5,86 & 30,41 & 30,97 & 79,10 & 66,88 & 8,58 & 38,49 & 39,44 & 77,51 \\
\hline & Desvio padrão & 2,03 & 0,53 & 1,06 & 1,09 & 0,85 & 2,31 & 1,55 & 3,37 & 3,61 & 1,21 \\
\hline \multirow[t]{2}{*}{ Cetol } & Média & 72,93 & 5,49 & 29,28 & 29,79 & 79,40 & 64,59 & 10,28 & 42,84 & 44,06 & 76,51 \\
\hline & Desvio padrão & 2,32 & 0,45 & 1,32 & 1,37 & 0,50 & 2,34 & 0,64 & 1,44 & 1,51 & 0,59 \\
\hline
\end{tabular}


com a madeira levando a formação deste. Já no caso do pau marfim houve um aumento da coordenada $b$ * para os dois tratamentos, inferindo que a aplicação dos produtos acentuou a pigmentação amarela, provavelmente devido a interação entre estes e a madeira.

Após as lâminas de madeira das duas espécies terem sido submetidas à ação do intemperismo pelos períodos estudados, foi constatada alteração de suas cores.

As Tabelas 3 e 4 apresentam os valores médios dos parâmetros colorimétricos para os três tratamentos e para duas espécies estudadas.
Observando-se os valores colorimétricos dos tratamentos submetidos a diferentes períodos de intemperismo para a espécie cumaru, houve uma diminuição da variável $L^{*}$ e aumento da coordenada a* no início dos tratamentos (até 40 horas). Depois de 76 horas de intemperismo, para os três tratamentos, a tendência foi para um aumento de $L^{*}$ e diminuição de $a^{*}$. É interessante observar que nos dois últimos estágios de intemperismo (838 e 960 horas), para os três tratamentos, inclusive a testemunha, os valores de $L^{*}$ foram maiores que o tempo inicial de tratamento. Isto é, a madeira ficou mais clara com o passar do tempo.

TABELA 3 Valores médios dos parâmetros colorimétricos de lâminas de cumaru para os três tratamentos e tempos de intemperismo estudados.

TABLE 3 Mean values of colorimetric parameters for cumaru wood blades for the three treatments and weathering times studied.

\begin{tabular}{|c|c|c|c|c|c|c|}
\hline Tratamento & Intemperismo (horas) & $L^{*}$ & $a^{*}$ & $b^{*}$ & $\mathrm{C}$ & $\mathrm{h}^{*}$ \\
\hline \multirow{10}{*}{$\begin{array}{c}1 \\
\text { Testemunha }\end{array}$} & 0 & $49,09 \mathrm{C}$ & $10,04 \mathrm{D}$ & $23,66 \mathrm{~A}$ & $25,70 \mathrm{~A}$ & $67,00 \mathrm{~B}$ \\
\hline & 20 & $27,13 F$ & $12,81 \mathrm{~A}$ & $16,70 \mathrm{E}$ & $21,05 \mathrm{D}$ & $52,41 \mathrm{~F}$ \\
\hline & 40 & $25,60 G$ & $11,89 \mathrm{~B}$ & $14,81 \mathrm{~F}$ & $19,00 \mathrm{E}$ & $51,17 G$ \\
\hline & 52 & $45,78 \mathrm{E}$ & $10,17 \mathrm{CD}$ & $22,84 \mathrm{AB}$ & $25,01 \mathrm{AB}$ & $66,00 \mathrm{CD}$ \\
\hline & 76 & $24,32 \mathrm{H}$ & $10,69 \mathrm{C}$ & $12,59 G$ & $16,52 \mathrm{G}$ & $49,60 \mathrm{H}$ \\
\hline & 124 & $47,00 \mathrm{D}$ & $10,30 \mathrm{CD}$ & $22,28 \mathrm{~B}$ & $24,55 \mathrm{~B}$ & $65,21 \mathrm{DE}$ \\
\hline & 226 & 47,92D & $9,82 \mathrm{D}$ & $21,12 \mathrm{C}$ & $23,29 \mathrm{C}$ & $65,10 \mathrm{E}$ \\
\hline & 430 & $49,01 \mathrm{C}$ & $8,45 \mathrm{E}$ & $19,23 \mathrm{D}$ & $21,02 \mathrm{D}$ & $66,46 \mathrm{BC}$ \\
\hline & 838 & $51,42 B$ & $6,34 \mathrm{~F}$ & $17,37 \mathrm{E}$ & $18,51 \mathrm{EF}$ & $70,33 \mathrm{~A}$ \\
\hline & 960 & $52,79 A$ & $6,12 \mathrm{~F}$ & $16,70 \mathrm{E}$ & $17,81 \mathrm{~F}$ & $70,40 A$ \\
\hline \multirow{10}{*}{$\begin{array}{c}2 \\
\text { Verniz Marítimo }\end{array}$} & 0 & $35,89 \mathrm{~F}$ & $13,17 \mathrm{~A}$ & $18,95 \mathrm{DE}$ & $23,08 \mathrm{AB}$ & $55,14 \mathrm{~F}$ \\
\hline & 20 & $30,92 \mathrm{G}$ & $10,87 \mathrm{C}$ & $12,45 \mathrm{~F}$ & $16,54 \mathrm{E}$ & $48,69 G$ \\
\hline & 40 & $28,69 \mathrm{H}$ & $10,04 \mathrm{D}$ & $11,51 \mathrm{G}$ & $15,54 \mathrm{~F}$ & $48,72 G$ \\
\hline & 52 & $38,17 \mathrm{E}$ & $12,06 \mathrm{~B}$ & $18,41 \mathrm{E}$ & $22,02 B$ & $56,73 E$ \\
\hline & 76 & $27,75 \mathrm{H}$ & $9,26 \mathrm{E}$ & $10,41 \mathrm{H}$ & $13,93 G$ & $48,31 \mathrm{G}$ \\
\hline & 124 & 42,79D & 10,37D & $19,42 \mathrm{CD}$ & $22,03 \mathrm{~B}$ & $61,92 \mathrm{D}$ \\
\hline & 226 & $47,68 \mathrm{C}$ & $8,73 F$ & $20,34 \mathrm{AB}$ & $22,16 \mathrm{~B}$ & $66,77 \mathrm{C}$ \\
\hline & 430 & $49,15 B$ & $6,62 \mathrm{G}$ & $19,86 \mathrm{BC}$ & $20,96 C D$ & $71,58 \mathrm{~B}$ \\
\hline & 838 & $51,27 \mathrm{~A}$ & $5,36 \mathrm{H}$ & $21,02 \mathrm{~A}$ & $21,71 \mathrm{BC}$ & $75,62 A$ \\
\hline & 960 & $51,54 \mathrm{~A}$ & $5,17 \mathrm{H}$ & $20,01 \mathrm{BC}$ & $20,68 \mathrm{D}$ & $75,44 \mathrm{~A}$ \\
\hline \multirow{10}{*}{$\begin{array}{c}3 \\
\text { Cetol }\end{array}$} & 0 & $42,18 \mathrm{D}$ & $11,19 \mathrm{D}$ & $21,71 \mathrm{BC}$ & $24,43 B$ & $63,25 \mathrm{BC}$ \\
\hline & 20 & $37,68 \mathrm{E}$ & $11,80 \mathrm{BC}$ & $18,97 \mathrm{E}$ & $22,37 \mathrm{C}$ & $57,82 \mathrm{G}$ \\
\hline & 40 & $36,11 \mathrm{~F}$ & $9,00 \mathrm{~F}$ & $14,81 \mathrm{~F}$ & $17,36 \mathrm{D}$ & $58,80 \mathrm{FG}$ \\
\hline & 52 & $42,15 \mathrm{D}$ & $12,19 \mathrm{AB}$ & $21,17 \mathrm{CD}$ & $24,44 \mathrm{~B}$ & $59,91 \mathrm{EF}$ \\
\hline & 76 & $37,38 \mathrm{EF}$ & $7,01 \mathrm{G}$ & $13,30 G$ & $15,12 \mathrm{E}$ & $62,18 \mathrm{CD}$ \\
\hline & 124 & $42,78 \mathrm{CD}$ & $12,47 \mathrm{~A}$ & $22,53 \mathrm{AB}$ & $25,75 \mathrm{~A}$ & $60,98 \mathrm{DE}$ \\
\hline & 226 & $44,08 \mathrm{BC}$ & $12,66 \mathrm{~A}$ & $23,03 \mathrm{~A}$ & $26,29 A$ & $61,14 \mathrm{DE}$ \\
\hline & 430 & $42,25 \mathrm{D}$ & $11,61 \mathrm{CD}$ & $20,54 \mathrm{D}$ & $23,60 \mathrm{~B}$ & $60,34 \mathrm{EF}$ \\
\hline & 838 & $45,09 A B$ & $10,53 E$ & $22,04 \mathrm{ABC}$ & $24,44 \mathrm{~B}$ & $64,39 \mathrm{AB}$ \\
\hline & 960 & $45,75 \mathrm{~A}$ & $10,24 \mathrm{E}$ & $22,13 \mathrm{ABC}$ & $24,40 \mathrm{~B}$ & $65,06 \mathrm{~A}$ \\
\hline
\end{tabular}

Os valores assinalados com a mesma letra, dentro de cada coluna, dos parâmetros colorimétricos, para cada tratamento, não diferem entre si ao nível de $5 \%$ de significância pelo Teste de Tukey. 
TABELA 4 Valores médios dos parâmetros colorimétricos de lâminas de pau marfim para os três tratamentos e tempos de intemperismo estudados. TABLE 4 Mean values of colorimetric parameters for pau marfim wood blades for the three treatments and weathering times studied.

\begin{tabular}{|c|c|c|c|c|c|c|}
\hline Tratamento & Intemperismo (horas) & $L^{*}$ & $a^{*}$ & $b^{*}$ & C & $h^{*}$ \\
\hline \multirow{10}{*}{$\begin{array}{c}1 \\
\text { Testemunha }\end{array}$} & 0 & $72,28 \mathrm{~B}$ & $5,80 G$ & $30,37 \mathrm{D}$ & $30,92 \mathrm{E}$ & $79,21 \mathrm{~A}$ \\
\hline & 20 & $54,11 \mathrm{G}$ & $11,87 \mathrm{~B}$ & $41,50 \mathrm{~A}$ & $43,18 \mathrm{~A}$ & $74,04 \mathrm{C}$ \\
\hline & 40 & $57,37 \mathrm{~F}$ & $11,23 \mathrm{C}$ & $38,32 \mathrm{~B}$ & $39,94 \mathrm{C}$ & $73,68 \mathrm{CD}$ \\
\hline & 52 & $67,20 \mathrm{E}$ & $10,36 \mathrm{D}$ & $34,02 \mathrm{C}$ & $35,56 \mathrm{D}$ & $73,08 \mathrm{DE}$ \\
\hline & 76 & $50,79 \mathrm{H}$ & $12,98 \mathrm{~A}$ & $39,44 \mathrm{~B}$ & $41,53 \mathrm{~B}$ & $71,79 G$ \\
\hline & 124 & $68,84 \mathrm{D}$ & $9,20 \mathrm{E}$ & $28,50 \mathrm{E}$ & $29,95 \mathrm{E}$ & $72,13 F G$ \\
\hline & 226 & $70,78 \mathrm{C}$ & $7,23 \mathrm{~F}$ & $21,83 F$ & $23,00 \mathrm{~F}$ & $72,80 G$ \\
\hline & 430 & $72,68 \mathrm{~B}$ & $4,47 \mathrm{H}$ & $14,08 \mathrm{G}$ & $14,78 \mathrm{G}$ & $76,55 \mathrm{EF}$ \\
\hline & 838 & $72,96 \mathrm{~B}$ & $2,88 \mathrm{I}$ & $11,69 \mathrm{H}$ & $12,05 \mathrm{H}$ & $77,28 \mathrm{~B}$ \\
\hline & 960 & $74,39 \mathrm{~A}$ & 2,641 & $11,23 \mathrm{H}$ & $11,55 \mathrm{H}$ & $70,40 \mathrm{~B}$ \\
\hline \multirow{10}{*}{$\begin{array}{c}2 \\
\text { Verniz Marítimo }\end{array}$} & 0 & $66,88 \mathrm{~B}$ & $8,58 \mathrm{~F}$ & $38,49 A B$ & $39,44 \mathrm{C}$ & $77,51 \mathrm{~B}$ \\
\hline & 20 & $54,96 \mathrm{~F}$ & $11,57 \mathrm{D}$ & $39,66 \mathrm{~A}$ & $41,62 \mathrm{~A}$ & $73,26 \mathrm{D}$ \\
\hline & 40 & $55,26 \mathrm{EF}$ & $12,08 \mathrm{CD}$ & $39,61 \mathrm{~A}$ & $41,42 \mathrm{AB}$ & $73,04 \mathrm{D}$ \\
\hline & 52 & $61,26 \mathrm{D}$ & $12,81 \mathrm{AB}$ & $39,67 A$ & $41,69 A$ & $72,11 \mathrm{E}$ \\
\hline & 76 & $56,50 \mathrm{E}$ & $13,03 \mathrm{~A}$ & $37,86 \mathrm{BC}$ & $40,04 \mathrm{BC}$ & $71,00 F$ \\
\hline & 124 & $61,91 \mathrm{D}$ & $12,32 \mathrm{BC}$ & $37,05 \mathrm{C}$ & $39,05 \mathrm{C}$ & $71,64 \mathrm{EF}$ \\
\hline & 226 & $64,03 \mathrm{C}$ & $10,16 \mathrm{E}$ & $33,06 \mathrm{D}$ & $34,59 \mathrm{D}$ & $72,99 \mathrm{D}$ \\
\hline & 430 & $66,24 \mathrm{~B}$ & $6,63 G$ & $26,12 \mathrm{E}$ & $26,97 \mathrm{E}$ & $76,03 \mathrm{C}$ \\
\hline & 838 & $69,90 A$ & $4,40 \mathrm{H}$ & $21,17 \mathrm{~F}$ & $21,63 F$ & $78,63 A$ \\
\hline & 960 & $69,06 \mathrm{~A}$ & $3,84 \mathrm{H}$ & $19,95 \mathrm{~F}$ & $20,33 F$ & $79,30 \mathrm{~A}$ \\
\hline \multirow{10}{*}{$\begin{array}{c}3 \\
\text { Cetol }\end{array}$} & 0 & $64,59 A$ & $10,28 \mathrm{D}$ & $42,84 \mathrm{~A}$ & $44,06 \mathrm{BC}$ & $76,51 \mathrm{~A}$ \\
\hline & 20 & $55,98 \mathrm{E}$ & $12,02 \mathrm{~B}$ & $40,03 C$ & $41,80 \mathrm{D}$ & $73,25 \mathrm{CD}$ \\
\hline & 40 & $54,56 \mathrm{~F}$ & $11,26 \mathrm{C}$ & $36,70 \mathrm{D}$ & $38,40 \mathrm{E}$ & $72,92 \mathrm{CD}$ \\
\hline & 52 & $62,02 \mathrm{~B}$ & $12,78 \mathrm{~A}$ & $43,30 \mathrm{~A}$ & $45,15 A$ & $73,56 \mathrm{BC}$ \\
\hline & 76 & $54,70 \mathrm{~F}$ & $12,00 \mathrm{~B}$ & 37,31D & $39,20 \mathrm{E}$ & $72,16 \mathrm{E}$ \\
\hline & 124 & $60,86 \mathrm{BC}$ & $13,27 A$ & $42,54 \mathrm{~A}$ & $44,57 \mathrm{AB}$ & $72,67 \mathrm{DE}$ \\
\hline & 226 & $60,37 \mathrm{C}$ & $12,99 \mathrm{~A}$ & $41,27 \mathrm{~B}$ & $43,27 \mathrm{C}$ & $72,52 \mathrm{DE}$ \\
\hline & 430 & $58,42 \mathrm{D}$ & $12,04 \mathrm{~B}$ & $37,28 \mathrm{D}$ & $39,19 \mathrm{E}$ & $72,15 \mathrm{E}$ \\
\hline & 838 & $58,78 \mathrm{D}$ & $10,27 D$ & $34,94 \mathrm{E}$ & $36,45 \mathrm{~F}$ & $73,65 \mathrm{BC}$ \\
\hline & 960 & $58,07 \mathrm{D}$ & $9,65 \mathrm{E}$ & $33,68 \mathrm{~F}$ & $35,08 \mathrm{G}$ & $74,03 B$ \\
\hline
\end{tabular}

Os valores assinalados com a mesma letra dentro de cada coluna dos parâmetros colorimétricos, para cada tratamento, não diferem entre si ao nível de $5 \%$ de significância pelo Teste de Tukey.

No caso dos tratamentos 2 e 3 a coordenada b* também mostrou tendência de aumentar nos últimos estágios de intemperismo, em relação ao estágio inicial. Isto pode ajudar a explicar a cor mais clara no final destes dois tratamentos para esta espécie. No entanto, devido a testemunha ter clareado ao final dos estágios de intemperismo, mostra que provavelmente esta espécie possui em sua constituição química, componentes que se oxidam com o intemperismo.

De uma forma geral, observa-se que entre os dois produtos de acabamentos, o verniz, ao final das fases de intemperismo mostrou um maior clareamento da madeira, com uma diminuição significativa da coordenada $a^{*}$ e um aumento significativo da coordenada $b^{*}$, em relação ao cetol. Por outro lado, o cetol mostrou uma maior estabilidade durante as fases de intemperismo, havendo uma maior uniformidade entre estes três parâmetros colorimétricos ( $L^{*}, \mathrm{a}^{*}$ e $\left.\mathrm{b}^{*}\right)$. Os valores de $h^{*}$, corroboram para esta observação, ficando em um intervalo entre 57 a 66 neste tratamento.

Os parâmetros colorimétricos das lâminas de madeira de pau marfim, após a aplicação dos três tratamentos e os 10 tempos de intemperismo podem ser vistos na Tabela 4. 
Observando-se a Tabela 4, as lâminas de madeira de pau marfim sem aplicação de produtos de acabamentos, mostrou tendência em estabilizar ao final dos períodos de intemperismo (838 e 960 horas), diminuindo os valores das coordenadas $a^{*}$ e $b^{*}$. Provavelmente, os constituintes químicos desta espécie tenham sofrido oxidação, levando a uma estabilização de sua cor ao final destes períodos mencionados.

O verniz marítimo, com o passar do tempo de intemperismo apresentou um clareamento para a espécie, com uma diminuição dos valores das coordenadas $a^{*}$ e b*. A saturação da cor também diminuiu, mostrando uma certa instabilidade do produto.

Para esta madeira, o cetol apresentou uma diminuição da claridade com o avanço do tempo de intemperismo, apresentando tendência de estabilizar a partir de 43 horas. Provavelmente o produto possui em sua composição elementos de cor mais escura e ou que interagem com os componentes da madeira levando a um escurecimento mais marcante das amostras que receberam este produto (GONÇALEZ et al, 2010). Também para esta espécie verifica-se uma tendência de maior uniformidade entre as variáveis colorimétricas a*e b*. Apesar da cromacidade ter diminuído em relação a testemunha, observa-se também uma tendência de estabilização nos períodos finais deste tratamento.

As Tabelas 5 e 6 apresentam a variação dos parâmetros colorimétricos das lâminas de madeiras das espécies estudadas, comparando os tratamentos I, 2 e 3. Onde $\Delta \mathrm{E}^{*}$ é a variação total da cor.
Analisando a Tabela 5, constata-se que a partir de valores negativos de $\Delta L^{*}$ houve escurecimento na superfície das lâminas de cumaru e, percebendo-se ainda que esse escurecimento foi mais representativo nas primeiras 76h. Segundo Hon (200l), o escurecimento inicial é atribuído à formação de compostos quinoidais em função, sobretudo, da degradação da lignina pela radiação UV na presença de oxigênio.

A Tabela 6 mostra também valores negativos de $\Delta L^{*}$ com escurecimento da superfície das lâminas de pau marfim, acentuando-se nas primeiras 76h.

Observa-se que tanto para a espécie de cumaru como a de pau marfim, a partir das 76h ocorre uma inversão da declividade do $\Delta L^{*}$ para os tratamentos I e 2 e depois de 430h para o tratamento 3, o que sugere o final do processo de escurecimento da madeira e, possivelmente, a estabilização.

Resultados semelhantes para o comportamento da variação de claridade foram encontrados em lâminas de pau marfim tratada com outros produtos de acabamento sob radiação UV por um período de 500h (LIMA et al., 20I3). Verifica-se ainda em estudos semelhantes que esse processo de clareamento com madeiras amarronzadas tende a estabilizar-se à medida que aumenta o tempo de intemperismo (SILVA; PASTORE, 2004; SILVA et al.,2007). Este é o caso do cumaru também observado neste estudo. Já madeiras mais claras, como é o caso do pau marfim tende ser mais difícil a estabilização de seus parâmetros colorimétricos.

TABELA 5 Variações dos valores dos parâmetros de cor de laminas de madeira de cumaru submetidos a diferentes tratamentos. TABLE 5 Variations of the color parameter values of Cumaru veneers subjected to different treatments.

\begin{tabular}{|c|c|c|c|c|c|c|c|c|c|c|}
\hline \multirow{2}{*}{ Parâmetro } & \multirow{2}{*}{ Tratamento } & \multicolumn{9}{|c|}{ Tempo de Intemperismo (horas) } \\
\hline & & 20 & 40 & 52 & 76 & 124 & 226 & 430 & 838 & 960 \\
\hline \multirow{3}{*}{$\Delta \mathrm{L}^{*}$} & 1 & $-21,97$ & $-1,52$ & 20,18 & $-21,46$ & 22,68 & 0,93 & 1,09 & 2,41 & 1,36 \\
\hline & 2 & $-4,97$ & $-2,23$ & 9,48 & $-10,43$ & 15,05 & 4,88 & 1,48 & 2,12 & 0,27 \\
\hline & 3 & $-4,50$ & $-1,57$ & 6,04 & $-4,77$ & 5,40 & 1,29 & $-1,82$ & 2,83 & 0,66 \\
\hline \multirow{3}{*}{$\Delta \mathrm{a} *$} & 1 & 2,77 & $-0,91$ & $-1,72$ & 0,52 & $-0,39$ & $-0,48$ & $-1,37$ & $-2,12$ & $-0,21$ \\
\hline & 2 & $-2,30$ & $-0,83$ & 2,01 & $-2,80$ & 1,12 & $-1,64$ & $-2,11$ & $-1,26$ & $-0,19$ \\
\hline & 3 & 0,62 & $-2,80$ & 3,19 & $-5,17$ & 5,45 & 1,29 & $-1,82$ & $-1,08$ & $-0,29$ \\
\hline \multirow{3}{*}{$\Delta \mathrm{b} *$} & 1 & $-6,96$ & $-1,89$ & 8,04 & $-10,25$ & 9,69 & $-1,17$ & $-1,88$ & $-1,86$ & $-0,67$ \\
\hline & 2 & $-6,49$ & $-0,94$ & 6,90 & $-8,00$ & 9,01 & 0,92 & $-0,48$ & 1,16 & $-1,01$ \\
\hline & 3 & $-2,74$ & $-4,15$ & 6,35 & $-7,87$ & 9,23 & 0,51 & $-2,50$ & 1,50 & 0,10 \\
\hline \multirow{3}{*}{$\Delta \mathrm{E}^{*}$} & 1 & 23,21 & 2,59 & 21,79 & 23,79 & 24,66 & 1,56 & 2,57 & 3,71 & 1,53 \\
\hline & 2 & 8,50 & 2,55 & 11,89 & 13,44 & 17,58 & 5,23 & 2,62 & 2,72 & 1,06 \\
\hline & 3 & 5,31 & 5,25 & 9,33 & 10,56 & 12,00 & 1,90 & 3,59 & 3,38 & 0,73 \\
\hline
\end{tabular}

Onde: 1 corresponde ao tratamento 1 (testemunha), 2 corresponde ao tratamento 2 (verniz marítimo) e 3 corresponde ao tratamento 3 (cetol). 
TABELA 6 Variações dos valores dos parâmetros de cor de laminas de madeira de pau marfim submetidos a diferentes tratamentos. TABLE 6 Variations of the color parameter values of pau marfim veneers subjected to different treatments.

\begin{tabular}{|c|c|c|c|c|c|c|c|c|c|c|}
\hline \multirow{2}{*}{ Parâmetro } & \multirow{2}{*}{ Tratamento } & \multicolumn{9}{|c|}{ Tempo de Intemperismo (horas) } \\
\hline & & 20 & 40 & 52 & 76 & 124 & 226 & 430 & 838 & 960 \\
\hline \multirow{3}{*}{$\Delta \mathrm{L}^{*}$} & 1 & $-18,17$ & 3,26 & 9,83 & $-16,41$ & 18,05 & 1,94 & 1,90 & 0,28 & 1,43 \\
\hline & 2 & $-11,91$ & 0,30 & 5,99 & $-4,75$ & 5,40 & 2,12 & 2,21 & 3,66 & $-0,83$ \\
\hline & 3 & $-8,61$ & $-1,42$ & 7,47 & $-7,33$ & 6,16 & $-0,48$ & $-1,96$ & 0,36 & $-0,71$ \\
\hline \multirow{3}{*}{$\Delta \mathrm{a}^{*}$} & 1 & 6,08 & $-0,64$ & $-0,87$ & 2,62 & $-3,77$ & $-1,97$ & $-2,76$ & $-1,59$ & $-0,24$ \\
\hline & 2 & 2,99 & 0,51 & 0,73 & 0,22 & $-0,71$ & $-2,16$ & $-3,52$ & $-2,24$ & $-0,55$ \\
\hline & 3 & 1,74 & $-0,76$ & 1,51 & $-0,78$ & 1,27 & $-0,48$ & $-1,96$ & $-1,77$ & $-0,62$ \\
\hline \multirow{3}{*}{$\Delta \mathrm{b}^{*}$} & 1 & 11,14 & $-3,18$ & $-4,30$ & 5,42 & $-10,94$ & $-6,67$ & $-7,75$ & $-2,39$ & $-0,45$ \\
\hline & 2 & 1,17 & $-0,05$ & 0,06 & $-1,82$ & $-0,81$ & $-3,99$ & $-6,94$ & $-4,95$ & $-1,22$ \\
\hline & 3 & $-2,81$ & $-3,33$ & 6,60 & $-5,99$ & 5,23 & $-1,27$ & $-3,98$ & $-2,34$ & $-1,26$ \\
\hline \multirow{3}{*}{$\Delta \mathrm{E}^{*}$} & 1 & 22,16 & 4,60 & 10,76 & 17,48 & 21,44 & 7,22 & 8,45 & 2,88 & 1,52 \\
\hline & 2 & 12,34 & 0,60 & 6,04 & 5,09 & 5,51 & 5,01 & 8,09 & 6,55 & 1,58 \\
\hline & 3 & 9,23 & 3,70 & 10,08 & 9,50 & 8,18 & 1,44 & 4,85 & 2,96 & 1,57 \\
\hline
\end{tabular}

Em que: 1 corresponde ao tratamento 1 (testemunha), 2 corresponde ao tratamento 2 (verniz marítimo) e 3 corresponde ao tratamento 3 (cetol).

A variação da cor total $\left(\Delta \mathrm{E}^{*}\right)$, Figura I, é maior para ambas as espécies até o tempo de intemperismo de 226 horas, Após este período há uma tendência de maior estabilização.

A variação total da cor fornece uma visão geral sobre desempenho das lâminas de madeira sob a ação do intemperismo. A variação da cor para o cumaru, no final do experimento ( $960 \mathrm{~h}$ ) para os produtos de acabamentos foi de I,06 e 0,73 para os tratamentos $2 \mathrm{e}$ 3 , sendo considerada, segunda a classificação de Hikita et al. (200I) como ligeiramente perceptível para ambos os tratamentos. Ainda segundo esta classificação, a variação da cor para o pau marfim, no final do experimento ( $960 \mathrm{~h}$ ) para os dois produtos de acabamentos foi de I,58 e I,57, classificando-as como notável. Novamente percebe-se uma maior estabilidade da espécie cumaru (madeira mais escura) com o passar do tempo de intemperismo, em relação ao pau marfim, madeira mais clara.

As reflectâncias das espécies de cumaru e pau marfim em função do comprimento de onda podem ser observadas nas figuras 2 e 3 , respectivamente. Apresentam-se curvas em intervalos de tempo de Oh, $226 \mathrm{~h}$ e $960 \mathrm{~h}$ nos três tratamentos analisados.

Considerando o espectro de luz visível (400-700 $\mathrm{nm}$ ), observa-se um formato padrão do comprimento de onda versus reflectância, tanto para o cumaru quanto para o pau marfim, submetidos aos três tratamentos. No entanto, verifica-se a inflexão das curvas diferente entre as espécies, mostrando o comportamento de cada uma de acordo com os tratamentos estudados. A madeira de cumaru quando comparada com a de pau marfim apresenta curvas de menores refletâncias para os três
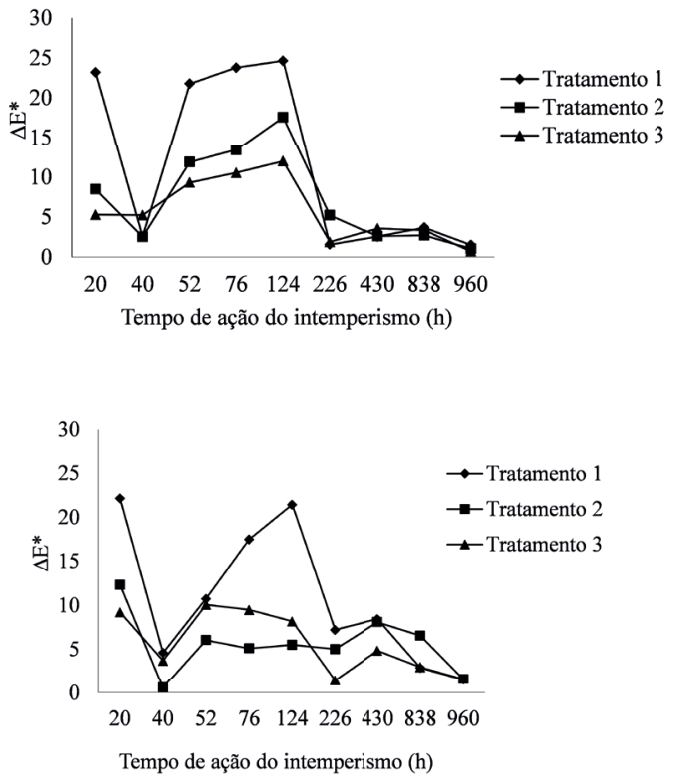

FIGURA 1 Variações dos parâmetros $\Delta \mathrm{E}^{*}$ provocadas pela ação do intemperismo em lâminas de madeira de cumaru e de pau marfim.

FIGURE 1 Variations of $\Delta \mathrm{E}^{*}$ caused by the action of weathering in veneers of cumaru and pau marfim.

tratamentos, confirmando os valores encontrados para os parâmetros colorimétricos. Tanto para o cumaru, quanto para o pau marfim, ○ tratamento 3 mostrou maior homogeneidade das curvas de reflectância, sugerindo que este produto consegue dar uma maior estabilização na cor da madeira durante os períodos de intemperismos estudados. 


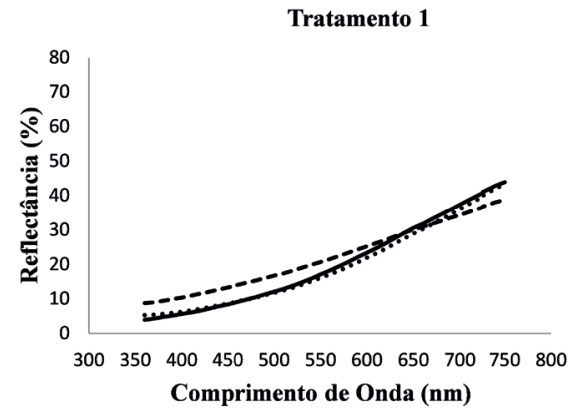

Tratamento 2

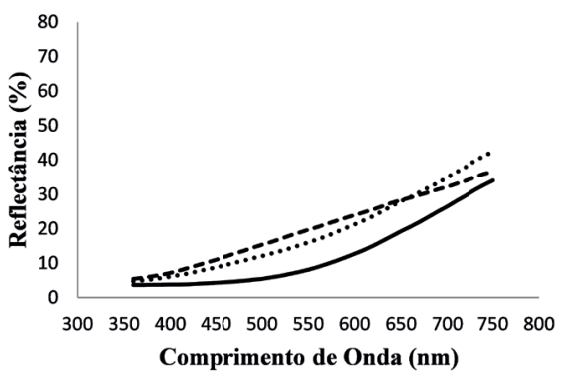

Tratamento 3

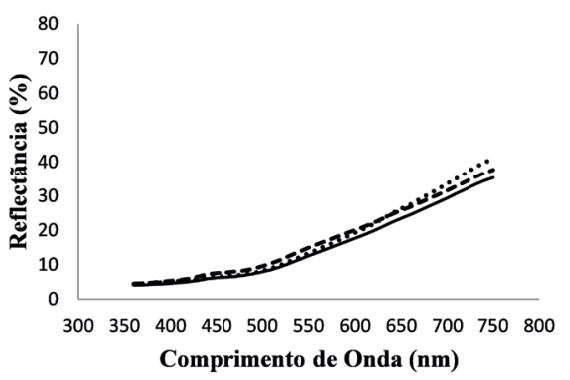

FIGURA 2 Reflectância do cumaru submetido a diferentes tratamentos em função do comprimento de onda.

FIGURE2 Reflectance of cumaru subjected to different treatments as a function of wavelength.

\section{CONCLUSÕES}

A espectrocolorimetria permitiu a obtenção de uma visão geral do comportamento das lâminas de madeira estudadas quando submetidas à ação do intemperismo.

A aplicação dos produtos de acabamento escureceu as lâminas de madeiras de cumaru e pau marfim em estado natural. No entanto, nos tempos de intemperismos mais elevados, ambas as espécies voltaram a clarear, chegando mesmo a ser superior os valores da claridade ao da madeira natural.

Os resultados da variação total da cor sugerem que tanto a madeira de cumaru como a de pau marfim apresenta menor variação da cor quando tratada com cetol. Sendo confirmado com as curvas de reflectância.

Recomenda-se um estudo dos componentes químicos de ambas as espécies, pois estes poderão

\section{Tratamento 1}

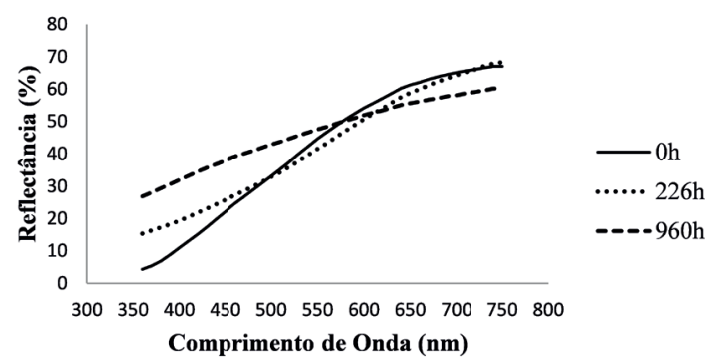

Tratamento 2
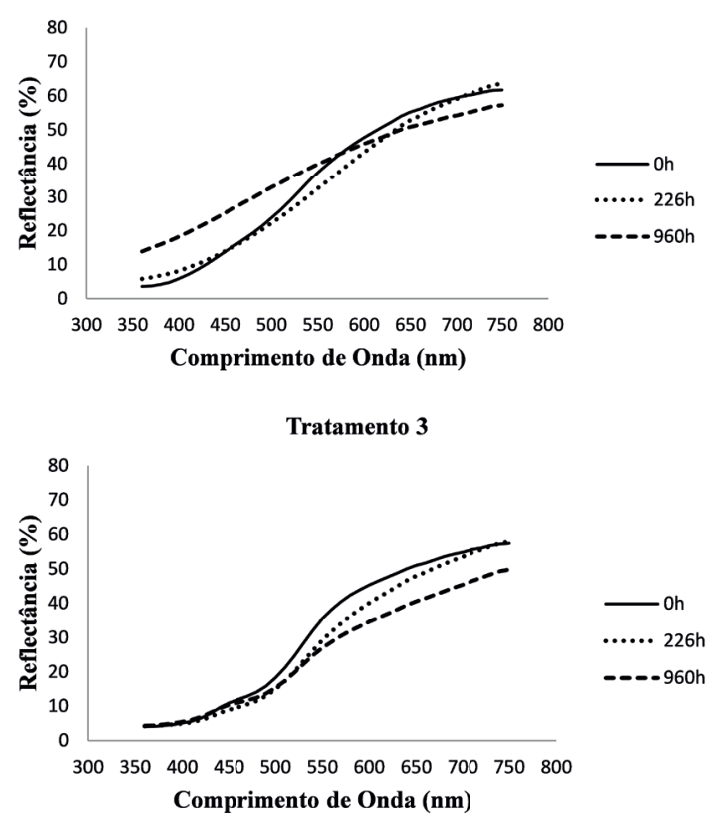

FIGURA 3 Reflectância do pau marfim submetido a diferentes tratamentos em função do comprimento de onda.

FIGURE 3 Reflectance of pau marfim subjected to different treatments as a function of wavelength.

dar mais subsídios aos fabricantes de produtos de acabamentos da madeira, no sentido de produzir produtos que estabilizam cada vez mais as madeiras frente ao intemperismo.

\section{REFERÊNCIAS}

GONÇALEZ, J. C. Caracterization technologique de quatre espèces peu connues de la forêt amazonienne: anatomie, chimie, couleur, propriétés physiques et mécaniques. 1993. $446 \mathrm{p}$. Thèse de Doctorat - Ministère de l'Agriculture et de la Pêche, Nancy, France, 1993.

GONÇALEZ, J.C.; FÉLIX, T.L.F.; GOUVEIA, F.N.; CAMARGOS, J.A.A.; RIBEIRO, P.G. Efeito da radiação ultravioleta na cor de madeira de Freijó (Cordia goeldiana Huber) após receber produtos de acabamentos. Ciência Florestal, Santa Maria, v.20, n.4, p.657-644, out-dez, 2010. 
GOUVEIA, F. N. Aplicação de tratamentos térmicos para estabilização colorimétrica de madeiras tropicais. Brasília: UnB, 2008. I 3 I p. Tese (Doutorado) - Programa de Pós-Graduação em Ciências Florestais, Faculdade de Tecnologia, Universidade de Brasília, Brasília, 2008.

HIKITA, Y.; TOYODA, T.; AZUMA, M. Weathering testing of timber: discoloration. In: IMAMURA, Y. High performance utilization of wood for outdoor uses. Kyoto: Press-Net, 2001.

HON, D.N.-S Weathering and photochemistry of wood. In: Hon, D.N.-S; Shiraishi,

N. Woos and cellulosic chemistry. 2.ed. New york: Marcel Dekker, 200I. 928p.
LIMA, C.M.; GONÇALEZ, J.C.; COSTA, T.R.V.da. PEREIRA, R.S. LIMA, J.B.M. LIMA, M.do S.A.L. Comportamento da Cor de Lâminas de Madeira de Pau-Marfim (Balfourodendron riedelianum) Tratada com Produtos de Acabamento. Revista Árvore, Viçosa-MG, v.37, n.2, p.377-384, 2013.

SILVA, J. O.; PASTORE, T. C. M. Fotodecomposição e proteção de madeiras tropicais.Floresta e Ambiente, Seropédica, v. II, n. 2, p. 07-13, 2004.

SILVA, J. O.; PASTORE, T. C. M.; PASTORE JÚNIOR, F. Resistência ao intemperismo artificial de cinco madeiras tropicais e de dois produtos de acabamento. Ciência Florestal, v. I7, n. I, p. I7-23, 2007.

SUDIYANI, Y.; TSUJIYAMA, S.; IMAMURA, Y.; TAKAHASHI, M.; MINATO, K.; KAlITA, H. Chemical characteristics of surfaces of hardwood and softwood deteriorated by weathering. Journal of Wood Science, v. 45, n. 4, p. 348-353, aug. 1999 
